\title{
Regulation of dendrite growth by Cdc42 effector protein-4 in hippocampal neurons in vitro
}

\author{
LEI HOU ${ }^{1,2^{*}}$, LUFENG WANG $^{3 *}$, ZHIJIE ZHAO ${ }^{4 *}$, WEI XU ${ }^{5}$, YANG WANG $^{6}$ and GANG CUI ${ }^{1}$ \\ ${ }^{1}$ Department of Neurosurgery, The First Affiliated Hospital of Soochow University, Suzhou, Jiangsu 215006; \\ ${ }^{2}$ Department of Neurosurgery, Jiading District Central Hospital Affiliated Shanghai University of Medicine and \\ Health Sciences, Shanghai 201899; ${ }^{3}$ Department of Neurology, Shanghai East Hospital, Tongji University School of Medicine, \\ Shanghai 200120; ${ }^{4}$ Department of Neurosurgery, Tongren Hospital, Shanghai Jiao Tong University School of Medicine, \\ Shanghai 200336; ${ }^{5}$ Department of Neurosurgery, Huashan Hospital, Fudan University, Shanghai 200040; \\ ${ }^{6}$ Department of Emergency, Xinhua Hospital, Shanghai Jiao Tong University School of Medicine, \\ Yangpu, Shanghai 200092, P.R. China
}

Received May 24, 2021; Accepted November 1, 2021

DOI: $10.3892 / \mathrm{mmr} .2022 .12644$

\begin{abstract}
Cell division control protein 42 homolog (Cdc42), one of the most characteristic members of the Rho protein family, is required for multiple aspects of dendritic morphogenesis. However, the proteins mediating the regulatory effects of Cdc42 activity on neuronal morphology are largely unknown. Cdc42 effector protein-4 (CEP4) was identified to be a binding partner of Rho GTPase 4 and is ubiquitously expressed in all adult tissues. However, the physiological function of CEP4 in neurons is unknown. In the present study, immunofluorescence and western blot analysis were conducted, revealing that CEP4 is highly expressed in the brain, and that the expression of CEP4 is gradually increased during neurodevelopment. Knockdown of CEP4 with short hairpin RNA suppressed dendrite growth, whereas overexpression of wild-type CEP4 promoted dendrite growth in primary isolated mouse hippocampal neurons. Collectively, these results indicated an important role for CEP4 in dendrite growth in hippocampal neurons.
\end{abstract}

Correspondence to: Dr Yang Wang, Department of Emergency, Xinhua Hospital, Shanghai Jiao Tong University School of Medicine, 1665 Kongjiang Road, Yangpu, Shanghai 200092, P.R. China E-mail: wang1989727@126.com

Professor Gang Cui, Department of Neurosurgery, The First Affiliated Hospital of Soochow University, 188 Shizi Street, Gusu, Suzhou, Jiangsu 215006, P.R. China

E-mail: CuiGang_2021@163.com

${ }^{*}$ Contributed equally

Key words: cell division control protein 42 homolog, cell division control protein 42 homolog effector protein-4, Rho GTPase, dendrite growth, hippocampal neurons

\section{Introduction}

The complex and characteristic structures of dendrites in neurons are critical to their function of receiving signals (1). Correct development of dendrites is essential for neuronal function, which depends on the coordinated organization of the cytoskeleton $(2,3)$. Rho GTPases are well-established regulators of the actin cytoskeleton in various biological processes, including cell migration, cell polarity and cell cycle progression (4). RhoA, Ras-related C4 botulinum toxin substrate 1 (Rac1) and cell division control protein 42 homologue (Cdc42) are extensively characterized members of the Rho family that control dendrite growth in mammalian neurons by regulating the growth dynamics of the cytoskeleton (5-8). To date, the most common view is that Rac1 and Cdc 42 positively regulate dendrite growth and dynamics, whereas RhoA acts as a negative regulator of dendrite arbor growth $(5,9,10)$.

$\mathrm{Cdc} 42$ is highly expressed in the nervous system and has emerged as a key regulator in dendrite branching and spine development and morphology (11-13). A previous report revealed that activated mutant $\mathrm{Cdc} 42$ expression inhibits dendritic arbor morphology in the Drosophila peripheral nervous system (14), while another study found that loss-of-function mutations of $\mathrm{Cdc} 42$ result in normal complexity but increased dendritic length, as well as defects in dendrite caliber and stereotyped dendritic branch positions in Drosophila mushroom body neurons, suggesting that $\mathrm{Cdc} 42$ is a regulator of multiple aspects of dendritic development (15). In Xenopus central neurons, it has been found that enhanced Cdc 42 activity selectively increased branch additions and retractions (16), and another study found that both dominant-negative and constitutively active $\mathrm{Cdc} 42$ decrease the total number of dendritic end tips (17). In mammals, dominant-negative $\mathrm{Cdc} 42$ expression in cortical neurons causes a marked reduction in the number of primary dendrites. Conversely, $\mathrm{Cdc} 42$ overexpression in cortical neurons leads to increased dendrite branching (10). However, one study reported that knockdown of Cdc42 expression with short hairpin RNA 
(shRNA) constructs increase dendritic branching in primary hippocampal neurons (18). Another study also demonstrated that inactivation $\mathrm{Cdc} 42$ by the GTPase-activating protein NOMA-GAP is necessary for dendritic branching in an analysis performed with transgenic mice (19). These contradictory reports suggest that the role of $\mathrm{Cdc} 42$ may vary in different types of neurons. However, the regulatory proteins of $\mathrm{Cdc} 42$ activity, such as $\mathrm{Cdc} 42$ effector protein, in regulating neuronal morphology are largely unknown.

Cdc42 effector protein-4 (CEP4), a binder of Rho GTPase-4, is ubiquitously expressed in all adult tissues $(20,21)$. It has been confirmed that $\mathrm{Cdc} 42$ binds to CEP4 at its 16 -amino acid $\mathrm{Cdc} 42 / \mathrm{Rac}$ interaction binding domain by site-specific mutagenesis (20). Previous studies have focused on CEP4's role in cancer (22-24), fibroblasts and epithelial cells (25). However, the physiological function of CEP4 in dendrite growth is unknown. In the present study, the expression of CEP4 in hippocampal neurons was investigated via western blotting and immunofluorescence (IF), and the role of CEP4 in dendrite growth was investigated by observing morphological changes in primary neurons following overexpression or knockdown of CEP4.

\section{Materials and methods}

Mice. A total of 8 pregnant ICR mice and 6 adult mice (age, 8-12 weeks; weight, 18-22 g) used in this study were purchased from the Shanghai SLAC Laboratory Animal Co., Ltd. The mice were bred and housed under standard conditions (temperature, $22 \pm 2{ }^{\circ} \mathrm{C}$; humidity, $50 \pm 5 \%$ ) with 12-h light/dark cycles and free access to standard food and water. All animal procedures were monitored by the Ethics Committee of the Soochow University and were performed according to the guidelines of the Animal Care Committee of Soochow University (approval no. SUDA20200116A01; Suzhou, China). The E18 embryos used in this study were sacrificed by decapitation with surgical scissors. The P1 and P3 neonatal pups were sacrificed by cervical dislocation following anesthetized with ice. The mice at P7, P14, P28, the pregnant females and adults were sacrificed by cervical dislocation following anesthesia with $4 \%$ isoflurane in $70 \% \mathrm{~N}_{2} \mathrm{O}$ and $30 \% \mathrm{O}_{2}$.

Plasmid construction. Total RNA was extracted from hippocampal cDNA of adult ICR mice using an RNeasy Mini Kit (Qiagen, Inc.). The first-strand cDNA was obtained using Superscript II reverse transcriptase (Vazyme Biotech Co., Ltd.) and reverse transcription was performed according to the manufacturer's protocol. The CEP4 was amplified by PCR using the following primers: Forward 5'-CAGCTCTGT GAACTCGAAGC-3' and reverse, 5'-GCTAGTGAGGAA AGACGTGTCC-3'. The following thermocycling conditions were used: $95^{\circ} \mathrm{C}$ for $3 \mathrm{~min}$; followed by 34 cycles of $95^{\circ} \mathrm{C}$ for $30 \mathrm{sec}, 55^{\circ} \mathrm{C}$ for $50 \mathrm{sec}$ and $72^{\circ} \mathrm{C}$ for $2 \mathrm{~min}$; and finally $72^{\circ} \mathrm{C}$ for $15 \mathrm{~min}$. The full-length CEP4 cDNA fragment was inserted into Plv-IRES-ZsGREEN1 plasmid [Jiman Biotechnology (Shanghai) Co., Ltd.]. The CEP4 shRNA sequences were as follows: shRNA1, 5'-CAGCACTGTTTG TCAAGAATG-3'; shRNA2, 5'-GGAAGATGAGATCCGAGT TTG-3'; shRNA3, 5'-CTCAGCTCAATGAGAAGGAAG-3'; and scramble shRNA (shSCR), 5'-TTCTCCGAACGTGTC
ACGT-3'. All shRNAs mentioned above were inserted into the pGMLV-SC5-EGFP vector [Jiman Biotechnology (Shanghai) Co., Ltd.].

HT22 cell culture and transfection. Mouse HT22 hippocampal neuronal cells were acquired from the Cell Bank of Shanghai Institute of Cells, Chinese Academy of Science. HT22 cells $\left(2 \times 10^{5} /\right.$ well) were seeded in a 6 -well culture plate and incubated in DMEM (Gibco, Thermo Fisher Scientific, Inc.) supplemented with 10\% FBS (Gibco; Thermo Fisher Scientific, Inc.) for $24 \mathrm{~h}$ prior to transfection. Subsequently, Lipofectamine ${ }^{\circledR} 3000$ transfection reagent (Invitrogen; Thermo Fisher Scientific) was used for plasmid transfection $(2,500 \mathrm{ng})$ according to the manufacturer's protocol. At 6-8 $\mathrm{h}$ post-transfection, the culture media was replaced with DMEM supplemented with $10 \%$ FBS and $100 \mathrm{U} / \mathrm{ml}$ penicillin/ streptomycin (Gibco; Thermo Fisher Scientific, Inc.). Cells were then grown for $48 \mathrm{~h}$ prior to harvesting.

Primary neuronal cell cultures and transfection. To obtain primary isolated hippocampal neurons from mice, E18 embryo brains were used; for each culture, one ICR pregnant female mouse was sacrificed and 5-6 E18 embryos were used. The E18 embryos were separated from the sacrificed nursing mother's uterus after the death of the pregnant mouse was confirmed. The hippocampus tissue was separated from the brain under a microscope and cut into small pieces. After centrifugation (194 x g, $5 \mathrm{~min}, 4^{\circ} \mathrm{C}$ ) to remove the supernatant, $1 \mathrm{ml}$ Papin (1 mg/ml; cat. no. p4752; Sigma-Aldrich; Merck KGaA) and DNase $(5 \mathrm{mg} / \mathrm{ml} ; 20 \mu \mathrm{l}$; cat. no. DN25; Sigma-Aldrich; Merck $\mathrm{KGaA}$ ) were added to the tube and incubated at $37^{\circ} \mathrm{C}$ for $30 \mathrm{~min}$. Subsequently, a single cell suspension was pipetted, centrifuged to remove the upper clear liquid and then added to complete medium. Isolated primary neurons $\left(5-10 \times 10^{4} / \mathrm{ml}\right)$ were seeded onto poly-D-lysine and cultured in Neurobasal-A medium (cat. no. 10888022; Gibco; Thermo Fisher Scientific, Inc.) supplemented with B27 (cat. no. 17504; Gibco; Thermo Fisher Scientific, Inc.), 1\% FBS (cat. no. 10099; Gibco; Thermo Fisher Scientific, Inc.) and penicillin/streptomycin mix (Gibco; Thermo Fisher Scientific, Inc.). For dendritic complexity, 7-day-old cultured hippocampal neurons in 24-well culture plates were transfected with different plasmids (shSCR, shCEP4-1 or 2; Vector or CEP4-HA; $500 \mathrm{ng} /$ well) for 7 days using the calcium phosphate precipitation method (26). For IF, 7-day-old cultured hippocampal neurons were transfected with the aforementioned plasmids for 5 days.

Western blotting. When analyzing the expression of CEP4 in the hippocampi from mice at different ages, 6 mice at P1, 6 mice at P3, 3 mice at P7, 3 mice at P14 and 3 mice at P28 were used in the present study. To detect the CEP4 expression in the central nervous system (CNS), the brain, spine, eye, optic nerve and different regions of brain were collected from mice following transcardial perfusion with cold PBS. Cultured HT22 cells, primary neurons [cultured 0 to 21 days in vitro (DIV)] and hippocampal regions from mice at different ages were harvested and lysed at $4^{\circ} \mathrm{C}$ in RIPA lysis buffer (cat. no. P0013; Beyotime Institute of Biotechnology) supplemented with $1 \mathrm{mM}$ PMSF and protease inhibitor cocktail (cat. no. 78425; Thermo Fisher Scientific, Inc.). Nuclear 
and cytosol proteins were obtained using nuclear extraction kit (cat. no. P0028; Beyotime Institute of Biotechnology). The protein concentrations were analyzed using a Bradford assay. Protein extracts were denatured for $5-8 \mathrm{~min}$ at $95^{\circ} \mathrm{C}$ in sample buffer. Protein extract $(20 \mu \mathrm{g})$ was loaded onto denatured $10 \%$ SDS gels (EpiZyme), then transferred onto PVDF membranes (MilliporeSigma). Membranes were subsequently blocked with $5 \%$ bovine serum albumin (Beyotime Institute of Biotechnology) for $1 \mathrm{~h}$ at room temperature and incubated with primary antibodies overnight at $4^{\circ} \mathrm{C}$. The following primary antibodies were used: Rabbit anti-CEP4 (1:1,000; cat. no. LS-C804922; Lifespan Biosciences); mouse anti-GAPDH (1:5,000; cat. no. G8795; Sigma-Aldrich; Merck $\mathrm{KGaA})$; rabbit anti- $\alpha$-tubulin $(1: 2,000$; cat. no. $2148 \mathrm{~S}$; Cell Signaling Technology, Inc.); mouse anti- HA Tag (1:5,000; cat. no. M20003; Abmart, Inc.); and rabbit anti-Lamin B (1:5,00; cat. no. AF1408; Beyotime Institute of Biotechnology). On the next day, the membranes were incubated with HRP-conjugated anti-mouse or anti-rabbit secondary antibodies (1:5,000; cat. nos. A0216 and A0208, respectively; Beyotime Institute of Biotechnology) at room temperature for $2 \mathrm{~h}$ following washing with TBST $(0.05 \%$ Tween). After washing three times with TBST, the HRP signals were detected with enhanced chemiluminescence reagents (Beyotime Institute of Biotechnology). Quantification was performed by analyzing the relative density of the immunoreactive bands using ImageJ software (version K 1.45; National Institutes of Health).

IF. Primary cultured neurons were fixed in $0.01 \mathrm{M}$ PBS ( $\mathrm{pH} 7.4$ ) containing $4 \%$ PFA for $30 \mathrm{~min}$ at $4^{\circ} \mathrm{C}$. For brain slices, $30 \mu \mathrm{m}$ coronal brain slices were generated following transcardial perfusion of mice with $4 \%$ PFA and fixed at $4^{\circ} \mathrm{C}$ overnight. The cells or brain slices were then blocked with blocking buffer $(0.3 \%$ Triton X-100 in PBS) containing $10 \%$ donkey serum (Jackson ImmunoResearch Laboratories, Inc.) for $1 \mathrm{~h}$ at room temperature. Then, the cells were incubated with primary antibodies in blocking buffer containing $2 \%$ goat serum overnight at $4^{\circ} \mathrm{C}$. The following primary antibodies were used for dual IF staining (same as used for western blotting if not stated otherwise): Anti-CEP4 (1:1,000 for cells, 1:200 for brain slices); chicken anti-microtubule-associated protein 2 (Map2; 1:10,000 for cells, 1:1,000 for slices; cat. no. MAP; Aves Labs); and mouse anti-HA $(1: 1,000)$. The cells or slices were then incubated with 488-conjugated donkey anti-rabbit antibody (cat. no. A-21206; Invitrogen; Thermo Fisher Scientific, Inc.), 555-conjugated donkey anti-rabbit antibody (cat. no. A-31572; Invitrogen; Thermo Fisher Scientific, Inc.), 647-conjugated donkey anti-mouse antibody (cat. no. A-31571; Invitrogen; Thermo Fisher Scientific, Inc.) or Cy3-conjugated donkey anti-chicken antibody (cat. no. 703-605-155; Jackson ImmunoResearch Laboratories, Inc.) at room temperature for $2 \mathrm{~h}$. Prior to visualization, the cells were incubated with DAPI (Invitrogen; Thermo Fisher Scientific, Inc.) for $5 \mathrm{~min}$ at room temperature, then washed with PBST. A confocal microscope (Leica SP8; Leica Microsystems GmbH) was used to capture images of cultured neurons and brain slices.

Image analysis and quantification. For the analysis of dendritic morphology, single neuronal images were obtained with a confocal microscope (magnification, x40; SP8; Leica
Microsystems $\mathrm{GmbH}$ ) and 6-8 fields of view were observed. Morphometric analysis and quantification were performed as previously described (27). Briefly, a z-series of 6-12 images with a $0.5-1 \mu \mathrm{m}$ depth interval was taken at 1,024x1,024-pixel resolutions. MetaMorph image analysis software version 7.1.3.0 (Molecular Devices, LLC) and the NeuronJ plugin in ImageJ was used to analyze and semi-quantify neuronal morphology as previously described (28-30). For Sholl analysis, a concentric circle with a diameter of $15 \mu \mathrm{m}$ was drawn around the cell body, and the number of dendrites passing through each circle was manually calculated.

Statistical analysis. Data are represented as the mean \pm SEM from at least three biological replicates for experiments. Statistical differences were determined by Student's t-test for two-group comparisons or ANOVA followed by Tukey's test for multiple comparisons among $>2$ groups. All data statistical analyses were performed using GraphPad Prism (version 8; GraphPad Software, Inc.).

\section{Results}

CEP4 is highly expressed in hippocampal neuron dendrites. Initially, CEP4 expression was investigated in CNS. As shown in Fig. 1A, CEP4 was highly expressed in the brain compared with its expression in the spine and optic nerve (Fig. 1A). To further explore the distribution of CEP4 in the brain, its expression pattern was analyzed via immunoblotting and IF. CEP4 was highly expressed in the cortex, hippocampus, thalamus and brainstem, while its expression in the cerebellum was substantially lower (Fig. 1B). Prominent labeling of CEP4 was also observed in the branching of neurons in the cortex, CA1, CA3 and the granule cell layer of the dentate gyrus (Fig. 1C). Expression of CEP4 was observed in cells positive for the neuronal marker Map2 both in dissociated cultures of hippocampal neurons and in hippocampal neurons in vivo, indicating that CEP4 is mainly expressed in the dendrites of neurons (Fig. 1D). To avoid the uncertainty of the results caused by polyclonal antibodies, an HA-tagged CEP4 plasmid was used to overexpress CEP4 in cultured neurons, which confirmed that CEP4 was mainly expressed in the cytoplasm and branching of neurons, as determined by IF staining with monoclonal anti-HA antibodies (Fig. 1E). Based on these findings, cytoplasmic and nuclear proteins were separately analyzed to determine the subcellular distribution of CEP4 both in vitro and in vivo. As shown in Fig. 1F, CEP4 was mainly expressed in the cytoplasm both in vitro and in vivo.

CEP4 is expressed in late developmental stages in dissociated cultures of hippocampal neurons and in the hippocampus. The expression of CEP4 was subsequently evaluated in dissociated cultures of hippocampal neurons and in mouse brains at various stages of development via western blotting; $\alpha$-tubulin was used as an internal standard. As shown in Fig. 2, CEP4 was present in dissociated hippocampal neurons from 0 to $21 \mathrm{DIV}$, displaying upregulation from day 5 onwards compared with day 1. In terms of CEP4 expression in vivo, CEP4 was detected in extracts from mouse hippocampus throughout development (P1 to P28), with increased expression at P7-28 compared with P1. 


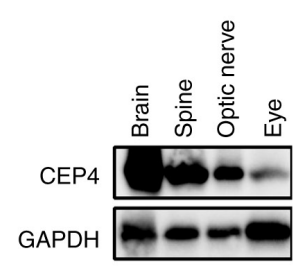

C

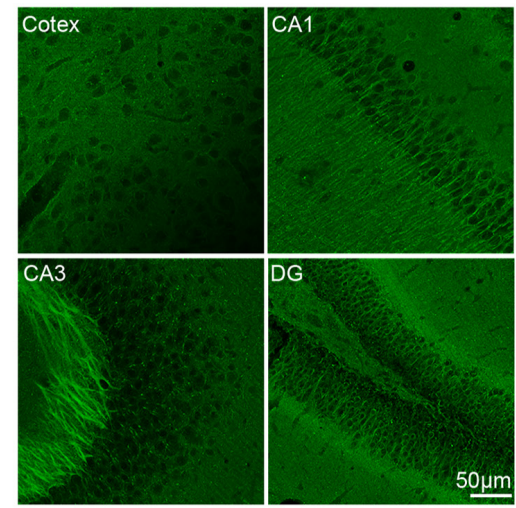

E

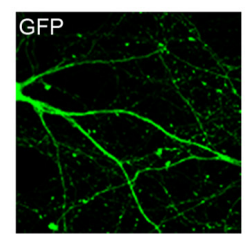

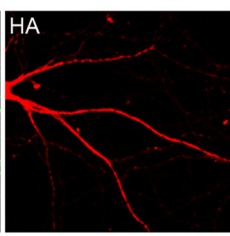

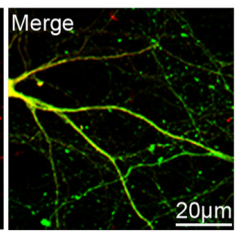

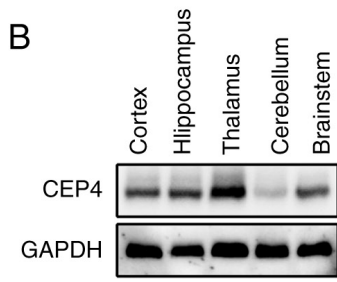

D
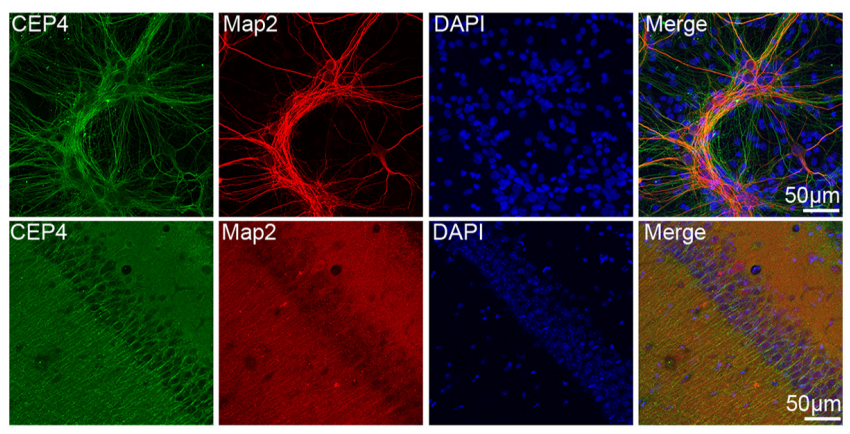

$\mathrm{F}$

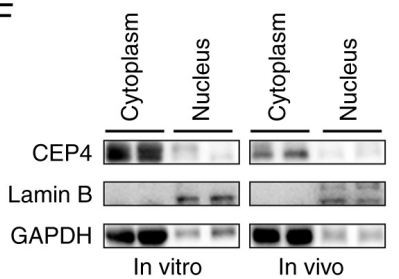

Figure 1. Identification of CEP4 expression in hippocampal neurons. Expression of CEP4 in (A) the central nervous system and (B) different regions of the brain was analyzed via western blotting. (C) Expression of CEP4 in the brain was analyzed via IF. (D) Co-expression of CEP4 (green) with the neuron dendritic marker Map2 (red) as determined via IF in brain slices and cultured hippocampal neurons after 14 DIV. (E) Dissociated cultures of hippocampal neurons transfected using the calcium phosphate method were stained with anti-HA antibodies to visualize the expression of CEP4 in neurons. (F) Nuclear and cytoplasmic expression of CEP4 in cultured hippocampal neurons at 14 DIV (in vitro) and in the hippocampus (in vivo) was detected via western blotting. CEP4, cell division control protein 42 homolog effector protein-4; Map2, microtubule-associated protein 2; IF, immunofluorescence; DIV, days in vitro; DG, dentate gyrus.

Knockdown of CEP4 using shRNA suppresses dendrite growth in primary cultured mouse hippocampal neurons. To investigate the neuronal function of CEP4, three shRNAs against CEP4 were designed: shCEP4-1, shCEP4-2 and shCEP4-3. CEP4 knockdown was first performed in HT22 cells. All the shRNAs induced efficient knockdown of CEP4, as determined via western blotting, with $>50 \%$ knockdown at the protein level, whereas shSCR had no effect on the expression of endogenous CEP4 (Fig. 3A). To further determine the effectiveness of the shCEP4 vectors, IF was performed using a specific antibody against CEP4. shCEP4-1 markedly decreased CEP4 protein level compared with shSCR-transfected cells (Fig. 3B). The effects of CEP4 knockdown on the dendritic arbor morphology of mouse hippocampal neurons were subsequently explored. Hippocampal neurons cultured for 7 DIV were used in this study, as intensive dendritogenesis occurs at this time point; shRNA plasmids were transfected into hippocampal neurons at 7 DIV, which were fixed at 14 DIV. Sholl analysis, which measures the number of dendrites crossing the circle at different radial distances from the cell soma, was used to quantify the branching pattern. In principle, a leftward and/or downward shift in Sholl analysis represents shrinkage of dendritic branching, whereas a rightward or upward shift
A
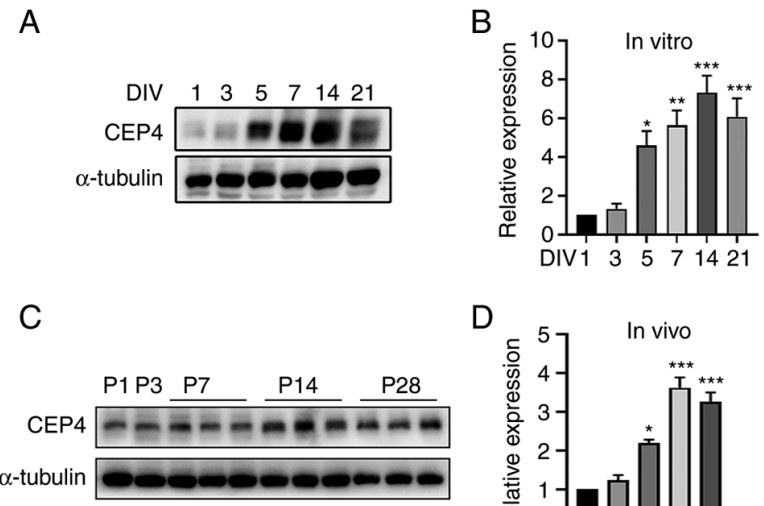

D

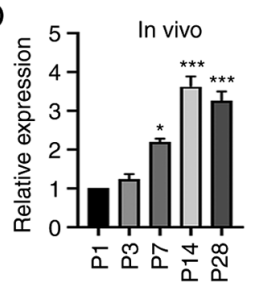

Figure 2. Expression of CEP4 increases during development. (A) Western blot analysis of CEP4 in primary dissociated cultured mouse hippocampal neurons. Lysates of dissociated cultures at various DIV were subjected to immunoblotting. (B) Quantification of CEP4 levels at different time points normalized to $\alpha$-tubulin ( $\mathrm{n}=3$ independent hippocampal cultures). ${ }^{*} \mathrm{P}<0.05,{ }^{* *} \mathrm{P}<0.01,{ }^{* * * *} \mathrm{P}<0.001$ vs. 1 DIV. (C) Western blot analysis of CEP4 in the mouse brain at various ages. Lysates of mouse brains at the indicated ages were subjected to immunoblotting. (D) Quantification of CEP4 levels at different time points normalized to $\alpha$-tubulin. Data are presented as the mean \pm SEM. ${ }^{*} \mathrm{P}<0.05,{ }^{*}{ }^{* * *} \mathrm{P}<0.001$ vs. $\mathrm{P} 1$. CEP4, cell division control protein 42 homolog effector protein-4; DIV, days in vitro. 

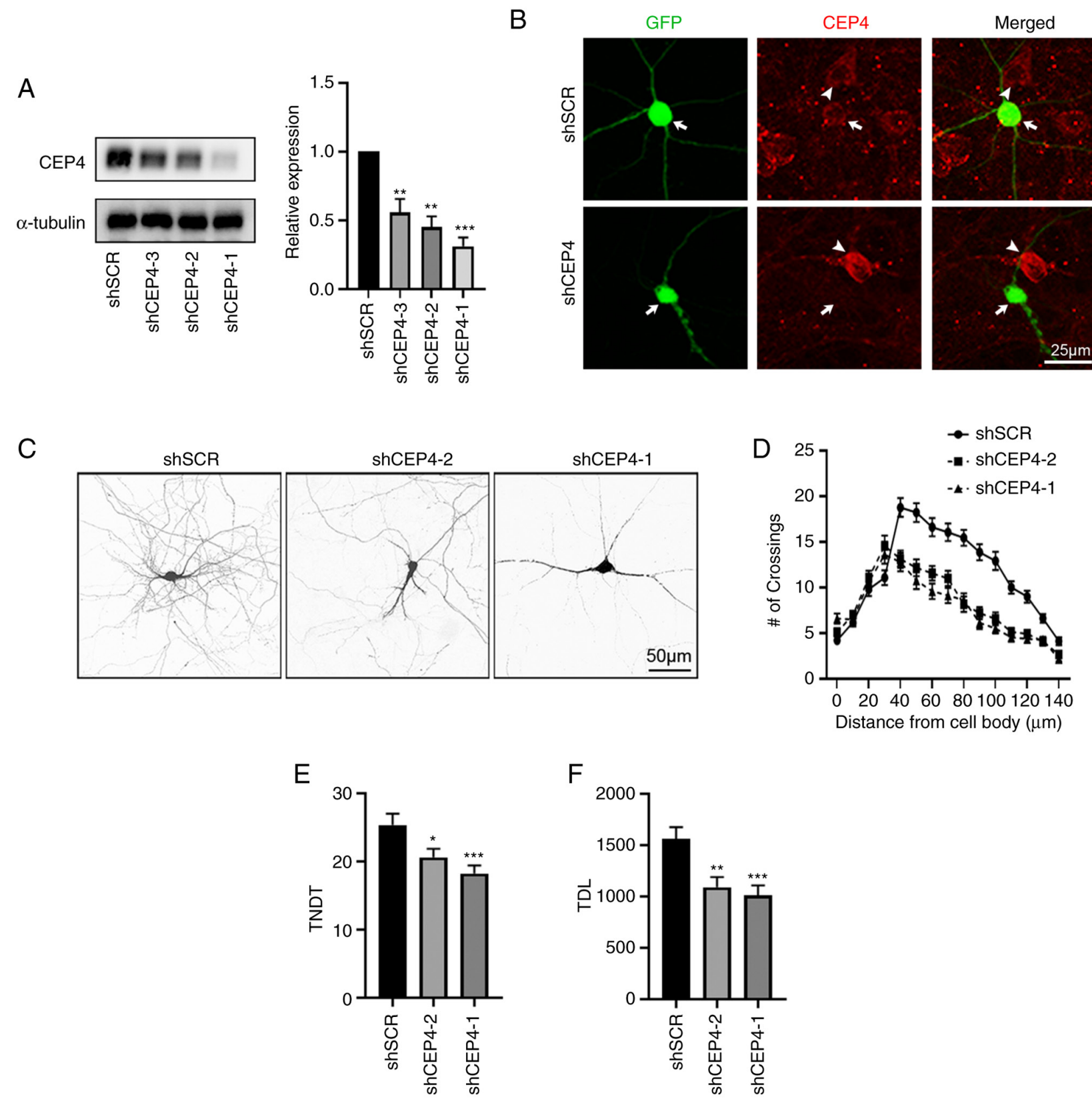

Figure 3. CEP4 knockdown in hippocampal neurons promotes the branching of dendrites. (A) HT22 cells were transfected with shSCR or shCEP4, and the silencing efficiency was identified via western blotting. The right panel shows quantification of immunoblotting. (B) Hippocampal neurons cultured in vitro were transfected on 7 DIV for 5 days with either shSCR or shCEP4-1. The cells were then stained with an antibody against CEP4; arrow indicates transfected neurons, whereas arrowheads indicate non-transfected cells. (C) Representative images of neurons transfected on 7 DIV for 7 days with shSCR, shCEP4-1 or shCEP4-2. (D) Sholl analysis of neurons transfected with shSCR, shCEP4-1 or shCEP4-2 (shSCR, n=48; shCEP4-1, n=48; shCEP4-2, n=48). (E) TNDT and (F) TDL of hippocampal neurons after CEP4 knockdown. Cell images were obtained from three culture batches. Data are presented as the mean \pm SEM. ${ }^{*} \mathrm{P}<0.05,{ }^{* *} \mathrm{P}<0.01,{ }^{* * *} \mathrm{P}<0.001$ vs. shSCR. CEP4, cell division control protein 42 homolog effector protein-4; TNDT, total number of dendritic tips; TDL, total dendrite length; sh, short hairpin; SCR, scramble; DIV, days in vitro.

represents increased complexity of the dendritic arbor. In the present study, a notable leftward and downward shift was observed in shCEP4-transfected hippocampal neurons compared with the shSCR group, indicating that CEP4 has a specific and important role in the dendrites of neurons (Fig. 3C and D). The introduction of shRNA against CEP4 markedly reduced the complexity of the dendritic arbors of transfected cells. Transfection with shCEP4-1 or shCEP4-2 significantly decreased the total number of dendritic tips (TNDT) compared with neurons transfected with shSCR (Fig. 3C and E). Changes in the total dendrite length (TDL) evoked by CEP4 knockdown were also evaluated. As presented in Fig. 3F, transfection of hippocampal neurons with CEP4 shRNA plasmids significantly decreased their TDL compared with shSCR. Taken together, these results indicated that knockdown of CEP4 in hippocampal neurons suppressed the growth of neuronal dendrites.

Overexpression of CEP4 in hippocampal neurons promotes dendrite branching. The finding that CEP4 knockdown in hippocampal neurons suppressed the growth of dendrites prompted speculation that CEP4 plays a positive regulatory 
A

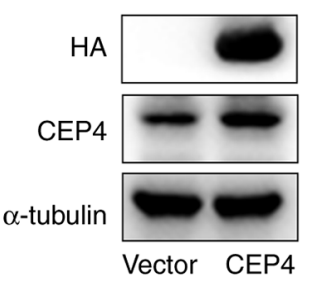

C

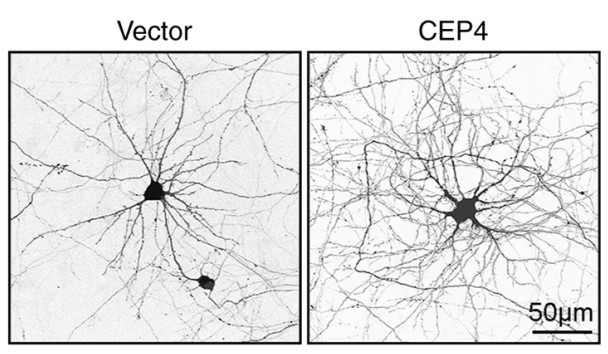

B

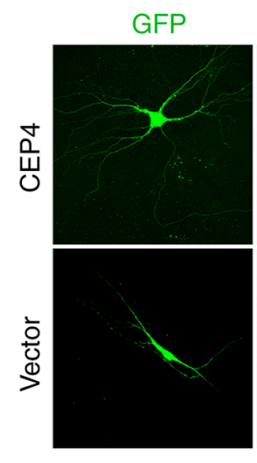

D

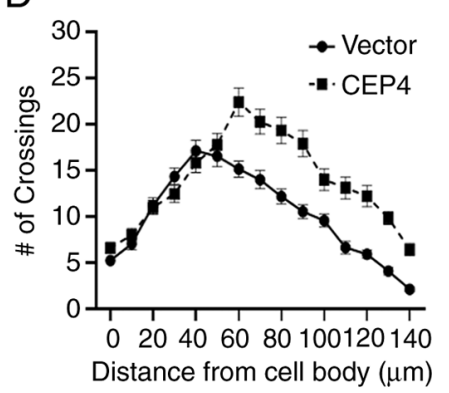

CEP4

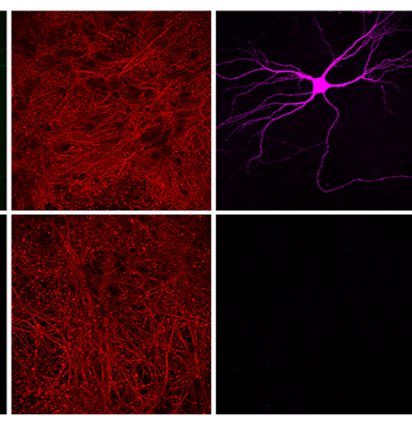

E

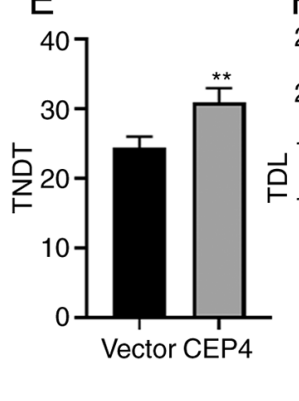

Merged

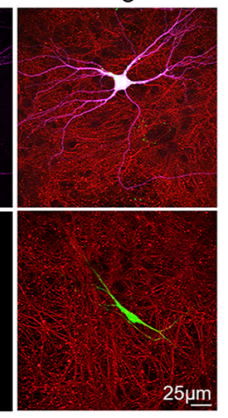

$\mathrm{F}$

Figure 4. CEP4 overexpression in hippocampal neurons promotes dendrite growth. (A) Overexpression of CEP4 in HT22 cells as determined via western blotting. (B) Hippocampal neurons cultured in vitro were transfected on 7 DIV for 5 days with control or CEP4-HA. The cells were subsequently stained with antibodies against CEP4 and HA. (C) Representative images of hippocampal neurons transfected on 7 DIV for 7 days with the control vector or CEP4 overexpression vector. (D) Sholl analysis of neurons transfected with the control vector or CEP4 overexpression vector (vector, $\mathrm{n}=48$; CEP4, $\mathrm{n}=48$ ). (E) TNDT and (F) TDL of hippocampal neurons transfected with the control vector or CEP4 overexpression vector. Cell images were obtained from three culture batches. Data are presented as the mean \pm SEM. ${ }^{* *} \mathrm{P}<0.01$ vs. vector. CEP4, cell division control protein 42 homolog effector protein-4; GFP, green fluorescent protein; TNDT, total number of dendritic tips; TDL, total dendrite length; DIV, days in vitro.

role in dendrite growth. Thus, a plasmid construct containing HA-tagged CEP4 cDNA was used to overexpress CEP4 in cultured hippocampal neurons. The overexpression of CEP4 in HT22 cells was confirmed via western blotting (Fig. 4A). In cultured hippocampal neurons, staining for CEP4 was not notably increased compared with the untransfected cells; however, staining for HA was only observed in CEP4-overexpressing cells (Fig. 4B). Contrary to the effect of CEP4 knockdown in neurons, CEP4 overexpression in cultures increased dendrite branching, TNDT and TDL (Fig. 4C). Sholl analysis showed that the peak of branching in neurons transfected with CEP4 was shifted rightward (farther from the soma) and upward (Fig. 4C and D). It was also observed that overexpression of CEP4 significantly increased the TNDT and TDL of neurons compared with neurons transfected with the empty vector (Fig. 4C, E and F). Taken together, these results indicated that CEP4 promoted the growth of dendrites in neurons.

\section{Discussion}

Numerous studies have demonstrated that altering the activity levels of Rho family proteins affects various aspects of dendritic development $(7,31,32)$. Cdc 42 , one of the most investigated members of the Rho family, has been found to act as a key regulator of neuronal morphology by controlling cytoskeletal reorganization (8). CEP4 is a Cdc42 effector protein that is distributed in various tissues, including the brain (21). Recently, one study found that knockdown of CEP4 resulted in an increase in the number of progenitors in the intermediate zone of the brain in a paired box protein 6-dependent manner (33); however, little is known concerning its role in dendrites in the hippocampus.

Binder of Rho GTPases (Borg)4, a member of the Borg family of proteins, is expressed ubiquitously in the brain, heart, kidneys, stomach and other tissues, as demonstrated via reverse transcription-quantitative PCR (21). Although some dissimilarity exists in the nucleotide sequences between CEP4 and Borg4, chromosomal localization supports the idea that CEP4 is an ortholog of mouse Borg4 (21). Thus, it is hypothesized that CEP4 is also widely expressed throughout these tissues. The presence of CEP4 in the brain supports the hypothesis that CEP4 is highly expressed in the CNS, particularly in the brain, as determined via western blotting in the present study. Further investigation explored the expression of CEP4 in specific brain regions. CEP4 was co-expressed with the neuronal dendritic marker Map2 in brain slices and isolated neurons, indicating that CEP4 is expressed in neurons. It was also found that CEP4 was highly expressed in the dendrites of neurons and the dendritic spines of hippocampal neurons by overexpressing the CEP4 sequence with a fusion HA tag in neurons. Furthermore, it was revealed that the expression of CEP4 remained high in cultured hippocampal neurons from 7 DIV and in vivo from $\mathrm{P} 7$, a key period during which dendrites grow and form a wide range of neuron-specific branching patterns to cover the target area (3). Thus, these results suggested that CEP4 may have important role in regulating dendrite growth. 
shRNA-mediated gene silencing is an important tool for biological research; these RNAs, through their hairpin-like structure, can recognize specific mRNAs and interfere with their degradation or hinder/inhibit their translation into proteins, ultimately achieving intervention at the mRNA and protein levels $(34,35)$. As knockdown efficiency varies for different shRNA target sites, it is necessary to design multiple shRNAs in the research process to screen the most effective knockdown vector (36). In the present study, three shRNA plasmids were designed for CEP4, and it was found that shCEP4-1 and shCEP4-2 induced effective knockdown of CEP4 based on protein levels. It was observed that knockdown of CEP4 significantly decreased TNDT and TDL, which suggested that CEP4 was necessary for dendrite growth. Conversely, overexpression of CEP4 increased TNDT and TDL, further supporting this hypothesis. Therefore, the present study revealed a potentially novel role for CEP4 in hippocampal neurons whereby CEP4 is necessary for dendritic morphogenesis.

Several limitations of the current study should be noted. First, rapid extension and arborization of dendrites occurs during the development of dendrites (37); therefore, the ability of CEP4 knockdown to inhibit the branching of dendrites may result from either the decreased formation of new branches or the increased retraction of existing branches. Further study is necessary to observe the development of living neurons with altered CEP4 expression on consecutive days using time-lapse microscopy. Second, changes in electrical potential following knockdown of CEP4 in hippocampal neurons have not been evaluated in the present study; electrical potential across the functional membrane is an important index of neuronal function (38). Thus, electrophysiological analysis should be conducted in future studies to evaluate the function of hippocampal neurons after CEP4 knockdown. Third, additional morphological or electrophysiological evaluations may be required to evaluate the effect of CEP4 knockdown or overexpression on dendritic spines in hippocampal neurons. Finally, the regulatory mechanism responsible for the effect of CEP4 on dendrite morphology remains unclear. Therefore, further elucidation of the related signaling pathways or interacting proteins of CEP4 is required.

In summary, to the best of our knowledge, this is the first study to elucidate the role of CEP4 in dendritic morphogenesis in the hippocampus in vitro. It was demonstrated that CEP4 was highly expressed in the brain, and that the expression of CEP4 was gradually upregulated during neuronal development. Combined with loss-of-function and gain-of-function analyses in primary cultured hippocampal neurons, the present study suggested that CEP4 is essential for dendritic growth.

\section{Acknowledgements}

Not applicable.

\section{Funding}

This study was supported by the National Natural Science Foundation of China for Young Scholars (grant no. 82102307) and the Biomedical and Engineering Cross Youth Fund of Shanghai Jiao Tong University (grant no. YG2021QN43).

\section{Availability of data and materials}

The datasets used and/or analyzed during the current study are available from the corresponding author on reasonable request.

\section{Authors' contributions}

GC and YW designed the study. LH, LW and ZZ performed the experiments and collected the data. LH, LW and WX interpreted the data and drafted the manuscript. GC, YW and WX reviewed and edited the manuscript. LH, LW, YW and GC confirmed the authenticity of all the raw data. All authors have read and approved the final manuscript.

\section{Ethics approval and consent to participate}

All animal procedures were monitored by the Ethics Committee of the Soochow University and conducted according to protocols approved by the Animal Care Committee of Soochow University (approval no. SUDA20200116A01).

\section{Patient consent for publication}

Not applicable.

\section{Competing interests}

The authors declare that they have no competing interests.

\section{References}

1. Takano T, Xu C, Funahashi Y, Namba T and Kaibuchi K: Neuronal polarization. Development 142: 2088-2093, 2015.

2. Muñoz-Lasso DC, Romá-Mateo C, Pallardó FV and Gonzalez-Cabo P: Much more than a scaffold: Cytoskeletal proteins in neurological disorders. Cells 9: 358, 2020.

3. Ledda F and Paratcha G: Mechanisms regulating dendritic arbor patterning. Cell Mol Life Sci 74: 4511-4537, 2017.

4. Hodge RG and Ridley AJ: Regulating Rho GTPases and their regulators. Nat Rev Mol Cell Biol 17: 496-510, 2016.

5. Chen $\mathrm{H}$ and Firestein BL: RhoA regulates dendrite branching in hippocampal neurons by decreasing cypin protein levels. J Neurosci 27: 8378-8386, 2007.

6. Leemhuis J, Boutillier S, Barth H, Feuerstein TJ, Brock C, Nürnberg B, Aktories K and Meyer DK: Rho GTPases and phosphoinositide 3-kinase organize formation of branched dendrites. J Biol Chem 279: 585-596, 2004.

7. Stankiewicz TR and Linseman DA: Rho family GTPases: Key players in neuronal development, neuronal survival, and neurodegeneration. Front Cell Neurosci 8: 314, 2014.

8. Chen C, Wirth A and Ponimaskin E: Cdc42: An important regulator of neuronal morphology. Int J Biochem Cell Biol 44: 447-451, 2012.

9. Scott GA and Cassidy L: Rac1 mediates dendrite formation in response to melanocyte stimulating hormone and ultraviolet light in a murine melanoma model. J Invest Dermatol 111: 243-250, 1998.

10. Threadgill R, Bobb K and Ghosh A: Regulation of dendritic growth and remodeling by Rho, Rac, and Cdc42. Neuron 19: 625-634, 1997.

11. Kalpachidou T, Spiecker L, Kress M and Quarta S: Rho GTPases in the physiology and pathophysiology of peripheral sensory neurons. Cells 8: 591, 2019.

12. Kamiyama D and Chiba A: Endogenous activation patterns of Cdc42 GTPase within Drosophila embryos. Science 324: 1338-1340, 2009.

13. Kreis P, ThévenotE, Rousseau V,Boda B, Muller D and Barnier JV: The p21-activated kinase 3 implicated in mental retardation regulates spine morphogenesis through a Cdc42-dependent pathway. J Biol Chem 282: 21497-21506, 2007. 
14. Luo L, Liao YJ, Jan LY and Jan YN: Distinct morphogenetic functions of similar small GTPases: Drosophila Drac1 is involved in axonal outgrowth and myoblast fusion. Genes Dev 8 : 1787-1802, 1994.

15. Scott EK, Reuter JE and Luo L: Small GTPase Cdc42 is required for multiple aspects of dendritic morphogenesis. J Neurosci 23: 3118-3123, 2003.

16. Li Z, Van Aelst L and Cline HT: Rho GTPases regulate distinct aspects of dendritic arbor growth in Xenopus central neurons in vivo. Nat Neurosci 3: 217-225, 2000.

17. Ruchhoeft ML, Ohnuma S, McNeill L, Holt CE and Harris WA The neuronal architecture of Xenopus retinal ganglion cells is sculpted by rho-family GTPases in vivo. J Neurosci 19 : 8454-8463, 1999.

18. Franke K, Otto W, Johannes S, Baumgart J, Nitsch R and Schumacher S: miR-124-regulated RhoG reduces neuronal process complexity via ELMO/Dock180/Rac1 and Cdc42 signalling. EMBO J 31: 2908-2921, 2012.

19. Rosário M, Schuster S, Jüttner R, Parthasarathy S, Tarabykin V and Birchmeier W: Neocortical dendritic complexity is controlled during development by NOMA-GAP-dependent inhibition of Cdc42 and activation of cofilin. Genes Dev 26: 1743-1757, 2012.

20. Joberty G, Perlungher RR and Macara IG: The Borgs, a new family of Cdc42 and TC10 GTPase-interacting proteins. Mol Cell Biol 19: 6585-6597, 1999.

21. Osada N, Kusuda J, Suzuki Y, Sugano S and Hashimoto K: Sequence analysis, gene expression, and chromosomal assignment of mouse Borg4 gene and its human orthologue. J Hum Genet 45: 374-377, 2000

22. Zhao X and Rotenberg SA: Phosphorylation of Cdc42 effector protein-4 (CEP4) by protein kinase $\mathrm{C}$ promotes motility of human breast cells. J Biol Chem 289: 25844-25854, 2014.

23. Chen X, Zhao X, Abeyweera TP and Rotenberg SA: Analysis of substrates of protein kinase $\mathrm{C}$ isoforms in human breast cells by the traceable kinase method. Biochemistry 51: 7087-7097, 2012.

24. Wangsa D, Chowdhury SA, Ryott M, Gertz EM, Elmberger G, Auer G, Lundqvist EÅ, Küffer S, Ströbel P, Schäffer AA, et al: Phylogenetic analysis of multiple FISH markers in oral tongue squamous cell carcinoma suggests that a diverse distribution of copy number changes is associated with poor prognosis. Int J Cancer 138: 98-109, 2016.

25. Hirsch DS, Pirone DM and Burbelo PD: A new family of Cdc 42 effector proteins, CEPs, function in fibroblast and epithelial cell shape changes. J Biol Chem 276: 875-883, 2001.

26. Passafaro M, Piëch V and Sheng M: Subunit-specific temporal and spatial patterns of AMPA receptor exocytosis in hippocampal neurons. Nat Neurosci 4: 917-926, 2001.
27. Chen K, Zhu L, Guo L, Pan YB and Feng DF: Maf1 regulates dendritic morphogenesis and influences learning and memory. Cell Death Dis 11: 606, 2020

28. Peng Y, Lee J, Rowland K, Wen Y, Hua H, Carlson N, Lavania S, Parrish JZ and Kim MD: Regulation of dendrite growth and maintenance by exocytosis. J Cell Sci 128: 4279-4292, 2015.

29. Jaworski J, Spangler S, Seeburg DP, Hoogenraad CC and Sheng M: Control of dendritic arborization by the phosphoinositide-3'-kinase-Akt-mammalian target of rapamycin pathway. J Neurosci 25: 11300-11312, 2005.

30. Swiech L, Blazejczyk M, Urbanska M, Pietruszka P, Dortland BR, Malik AR, Wulf PS, Hoogenraad CC and Jaworski J: CLIP-170 and IQGAP1 cooperatively regulate dendrite morphology. J Neurosci 31: 4555-4568, 2011.

31. Van Aelst L and Cline HT: Rho GTPases and activity-dependent dendrite development. Curr Opin Neurobiol 14: 297-304, 2004.

32. Chan WWR, Li W, Chang RCC and Lau KF: ARF6-Rac1 signaling-mediated neurite outgrowth is potentiated by the neuronal adaptor FE65 through orchestrating ARF6 and ELMO1. FASEB J 34: 16397-16413, 2020.

33. Narayanan R, Pham L, Kerimoglu C, Watanabe $T$, Hernandez RC, Sokpor G, Ulmke PA, Kiszka KA, Tonchev AB Rosenbusch J, et al: Chromatin remodeling BAF155 subunit regulates the genesis of basal progenitors in developing cortex. iScience 4: 109-126, 2018.

34. Rao DD, Vorhies JS, Senzer N and Nemunaitis J: siRNA vs. shRNA: Similarities and differences. Adv Drug Deliv Rev 61: 746-759, 2009

35. Moazed D: Small RNAs in transcriptional gene silencing and genome defence. Nature 457: 413-420, 2009.

36. Boettcher M and McManus MT: Choosing the right tool for the job: RNAi, TALEN, or CRISPR. Mol Cell 58: 575-585, 2015.

37. Williams DW and Truman JW: Mechanisms of dendritic elaboration of sensory neurons in Drosophila: Insights from in vivo time lapse. J Neurosci 24: 1541-1550, 2004

38. Li Y, Li X, Wang H, Gao Q, Zhang J, Zhang W, Zhang Z, Li L, Yu Y and Shuai L: CRISPR/Cas9-edited Pax6-GFP reporter system facilitates the generation of mouse neural progenitor cells during differentiation. J Genet Genomics 45: 277-280, 2018.

(7) $\odot$ This work is licensed under a Creative Commons Attribution-NonCommercial-NoDerivatives 4.0 International (CC BY-NC-ND 4.0) License. 